\title{
Expression of Endothelin-1 in Human Placenta of Active Smokers: An Immunohistochemical Study
}

\author{
Banajit Bastia $^{1}$, Kapil Kumar ${ }^{2}$, Shashi Nandar Kumar ${ }^{1}$, Nihar Ranjan Behera ${ }^{3}$, \\ Arun Kumar Jain ${ }^{1 *}$ \\ ${ }^{l}$ Environmental Toxicology \& Electron Microscope Lab, ICMR-National Institute of Pathology, Safdarjung \\ Hospital Campus, New Delhi, India \\ ${ }^{2}$ Department of Zoology, Meerut College, Meerut (C.C.S University), U.P, India \\ ${ }^{3}$ Department of Obstetrics \& Gynaecology, M.K.C.G Medical College \& Hospital, Berhampur, Odisha, India \\ *Corresponding Author: Arun Kumar Jain, Environmental Toxicology \& Electron Microscope Lab, \\ ICMR-National Institute of Pathology, Safdariung Hospital Campus, New Delhi, India
}

\begin{abstract}
Endothelin-1 (ET-1) is the most potent vasoconstrictor peptide secreted by placenta. Its localization in placental tissue of smoking women has been scarcely reported. In this study, ET-1 expression was examined and compared with that of CD-31 (endothelial cell marker) in the placenta of non-smoking and active smoking women using Avidin-Biotin-Complex (ABC) developer kit. ET-1 immunoreactivity was widely distributed in the small and medium sized capillaries and venules in placental villi of both the groups. The distribution of ET-1 in placental villi was similar to the distribution of CD-31. It was finely granular, dark brown in color and the product of immunoreaction was localized in the cytoplasm of endothelial cells lining the capillaries of placental villi. Specific and high affinity binding sites for ET-1 in placental endothelial cells were noticed in both the groups. No difference was discernible in the distribution pattern of ET-1 in placental villi between smokers and non-smokers group but the ET-1 immunoreactivity in placenta of active smokers was consistently observed to be more intense as compared to non-smokers' placenta. Thus, it is thought that exposure to tobacco during pregnancy may influence the production of ET-1 by endothelial cells in human placenta. Consequently, it is presumed that during pregnancy, the production of ET-1 may be upregulated in the placenta of active smokers which might be responsible for the adverse outcomes of pregnancy due to tobacco exposure.
\end{abstract}

Keywords: Endothelin-1, Endothelial cell marker (CD-31), Endothelial cell, Immuno-staining, Placenta, Smoker.

\section{INTRODUCTION}

Smoking during pregnancy is very well acknowledged to increase the risk of vascular complications in otherwise low-risk women (Quinton et al., 2008). Smoking also increases the risk of preterm birth, placental abruption and placenta praevia in uncomplicated pregnancies. Prenatal exposure to smoking in pregnant women increases the risk of delivering babies with Low Birth-weight (LBW), intrauterine growth restriction (IUGR) (Huang et al., 2017) and causes perinatal morbidity and mortality (Salafia and Shiverick, 1999; Quinton et al., 2008). The placenta is a highly specialized organ that supports the exchange of nutrients and waste products between maternal and fetal circulatory system. In addition, placenta secretes a large number of hormones and peptides, which help in regulation of feto-placental circulation, maternal health and fetal outcome (Gude et al., 2004). In normal pregnancy, the placental vasculature is dilated to its maximum capacity to permit optimal exchange between mother and the fetus. Towards the end of pregnancy, stimulation of local autacoids enhances vasoconstriction as well as vasodilation leading to normal delivery of the baby. Long acting autacoids such as endothelins have been implicated for mediating sustained vasoconstriction (To, 2012; Carter and Myatt, 1995). Among these, endothelins are recently discovered endothelium derived peptides, which show potent contractile effects in vascular and visceral smooth muscles. Out of the three different isoforms (ET-1, ET-2 and ET-3), Endothelin-1 (ET-1) is the most potent vasoconstrictor compound (Masaki and Yanagisawa, 1992). It is a peptide having 21 amino acids, which was first isolated and sequenced from the supernatant of cultured porcine aortic endothelial cells (Yanagisawa et al., 1988). The effects 
of ET-1 have been extensively studied in the normal placental tissue as well as maternal serum (Gude et al. 1991; Myatt et al., 1992). The levels of ET-1 have been reported to be elevated in maternal serum in patients with Pregnancy Induced Hypertension (PIH) and Pre-eclampsia (Sudo et al., 1993). It is also reported that ET-1 is localized in endothelial cells (Marinoni et al., 1995) of chorionic villi, amniotic epithelium (Hemsen et al., 1991) and villous trophoblast (Marinoni et al., 1995; Malassine et al., 1993) of human placenta. But the localization of ET-1 in the placental tissue of smoking mother is very sparsely studied. According to Economides and Braithwaite (1994) nicotine in any form increases the level of carbon monoxide in blood leading to inactivation of fetal and maternal hemoglobin and thus resulting in hypoxia. Asmussen (1980) and Jauniaux and Burton (1992) reported morphological and ultrastructural changes in placenta of smoking mother but very few literature is available on localization of ET-1 in smoker's placenta. Therefore, the present study was undertaken to examine the distribution pattern and localization of ET-1 in tobacco smokers in comparison to nonsmokers with the help of immunohistochemical technique.

\section{MATERIALS AND METHODS}

\subsection{Sample Collection}

Placentas $(n=50)$ were collected immediately after normal singleton vaginal delivery from healthy full-term mothers who had been previously enrolled for this study visiting the Antenatal Clinic (ANC) and admitted in Labor Room of Dept. of Obstetrics \& Gynecology, MKCG Medical College and Hospital, Berhampur, Odisha, India. The participants were classified into two groups ( $\mathrm{n}=25$ each) based on the tobacco exposure before and during pregnancy. Non-smokers (Control Group) were those who reported no history of smoking either by herself or any of her close relatives. Active Smokers were those mothers who directly smoked 15 cigarettes or 30 'bidis' or more per day for at least 2 years before and during pregnancy.

Subjects were matched for maternal age, parity and gestational age. Those with history of any debilitating diseases were excluded from our study. For immuno-histochemical studies, the placental tissues were collected from the fetal side of placenta after fixed by perfusion technique in Trump's fixative ( $\mathrm{pH}$ 7.2-7.4). All placentas were examined for conventional gross and light microscopic features as per the guidelines of the College of American Pathologists (Driscoll and Langston, 1991). The study was approved by the local Scientific Ethical Committee and samples were collected according to the "Ethical guidelines for biomedical research on human participants" issued be Indian Council of Medical Research (ICMR).

\subsection{Tissue Processing}

$5 \mathrm{~mm}$ placental tissue sections were immersion fixed in $10 \%$ buffered formalin within 10 min after delivery. The fixed placental tissues were processed for routine paraffin embedding. Five micron $(5 \mu \mathrm{m})$ thick sections were cut and mounted on clean glass slides.

\subsection{Immunohistochemistry}

These slides were kept in the oven at $60^{\circ} \mathrm{C}$ for 20 minutes. Tissue sections were then de-paraffinized and rehydrated. The endogenous peroxidase activity was quenched by immersing the de-paraffinized sections in $3 \% \mathrm{H}_{2} \mathrm{O}_{2}$ in Methanol for 30 minutes. Sections were rinsed in Phosphate Buffered Saline (PBS) of pH-7.2 and then exposed to microwaves for 10 minutes in Antigen Retrieval Solution (8.2 $\mathrm{mM}$ sodium citrate, $1.8 \mathrm{mM}$ Citric acid-pH-6.0, containing $0.01 \%$ Triton X-100). After subsequent washing with PBS, the sections were exposed to blocking serum for 45 minutes to block the nonspecific binding. The sections were then incubated in a humidified chamber $\left(37^{\circ} \mathrm{C}\right)$ with primary monoclonal anti-Endothelin-1 antibody (Oncogene Research Products, Boston, MA, USA) at a dilution of 1:250 (tested over a range of 1:100 to 1:1000) diluted in PBS containing 1\% BSA and $0.2 \%$ Tween-20 for overnight at $4-8^{\circ} \mathrm{C}$. Negative controls were achieved by omitting primary antibody while rest of the procedure remained the same.

The sections were then washed in PBS containing 0.2\% Tween-20 and immuno-staining was achieved using an Avidin-Biotin Complex (ABC) developer kit (Vectastain Elite ABC Kit, Vector Laboratories; Burlingame, CA, USA). The complex was detected using 3, 3'-diaminobenzidine (DAB) as a substrate. Sections were counterstained with Mayer's hematoxylin for 2 minutes, washed and mounted (Hsu et al., 1981). In each experiment, an additional serial section was stained with anti- 
Human CD-31 mAb (pre-diluted concentration of $200 \mu \mathrm{g} / \mathrm{ml}$; Sigma, St. Louis, MO, USA) that served as a positive control and permitted a comparison between the distribution of ET-1 and that of the endothelial cell marker, CD-31. Olympus upright microscope fitted with digital camera (Olympus, E520) was used for capturing microphotographs.

\section{RESUltS}

Endothelial cells (EC) were identified and confirmed by immunostaining with endothelial cell marker, anti-human CD-31 mAb. Fine granular and dark brown deposits of ET-1 (achieved due to DAB substrate \& chromogen reaction) were observed in the ECs lining the capillaries of tertiary villi of full-term control placenta (Fig.1). Immunostaining was limited to ECs only and was not discernible in syncytiotrophoblasts or other cells of placenta. The distribution pattern of ET-1 was similar to the immunostaining of endothelial cell marker, anti-human CD-31 (Fig.2). This confirms that the expression and localization of ET-1 is only in the endothelial cells of placental villi.

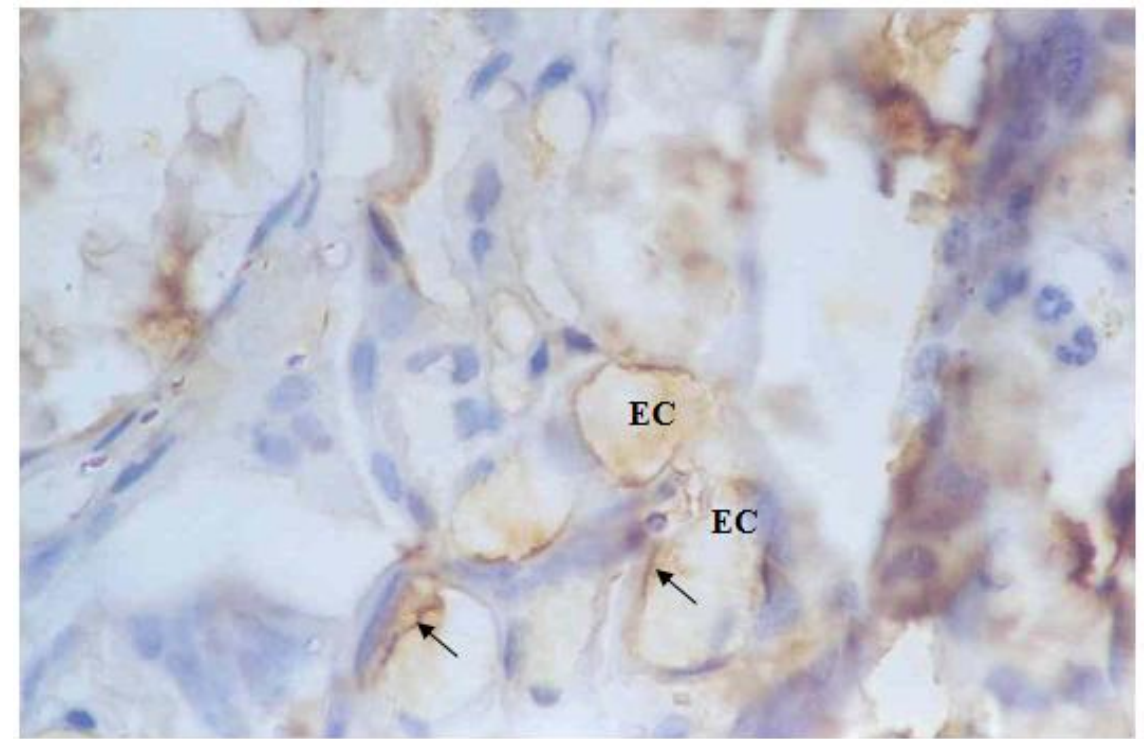

Fig1. Endothelial cells (EC) in capillaries of chorionic villi of placenta characterized by ET-1 (arrow) positivity. (Non-smoker, ET-1 mAb, Mayer's Haematoxylin, 100x)

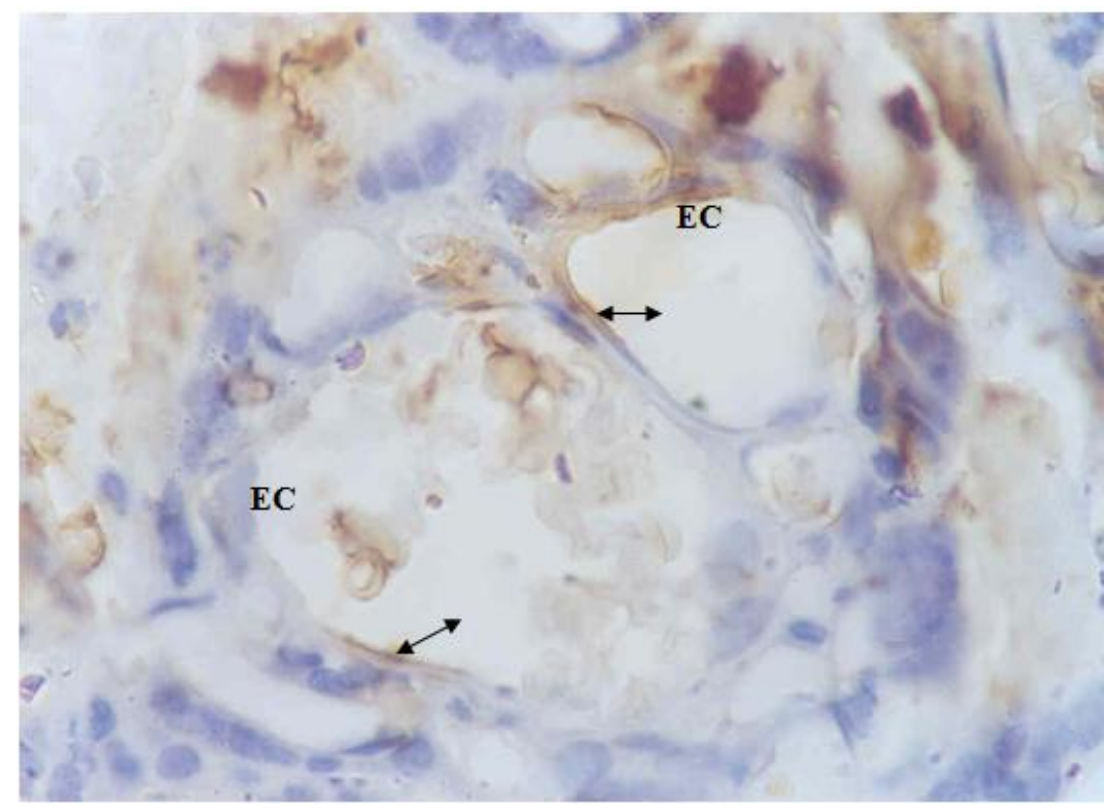

Fig2. CD-31 immunoreactivity (double arrow) in EC of placental capillaries is localized to similar regions showing ET-1 positivity in Fig.1. (Non-smoker, CD-31 mAb, 100x)

Negative control (Fig. 3 a \& b) shows absence of immunostaining due to omission of primary antibody from the normal Avidin-Biotin complex (ABC) procedure indicating thereby that the staining is specific for ET-1 as well as CD-31. 

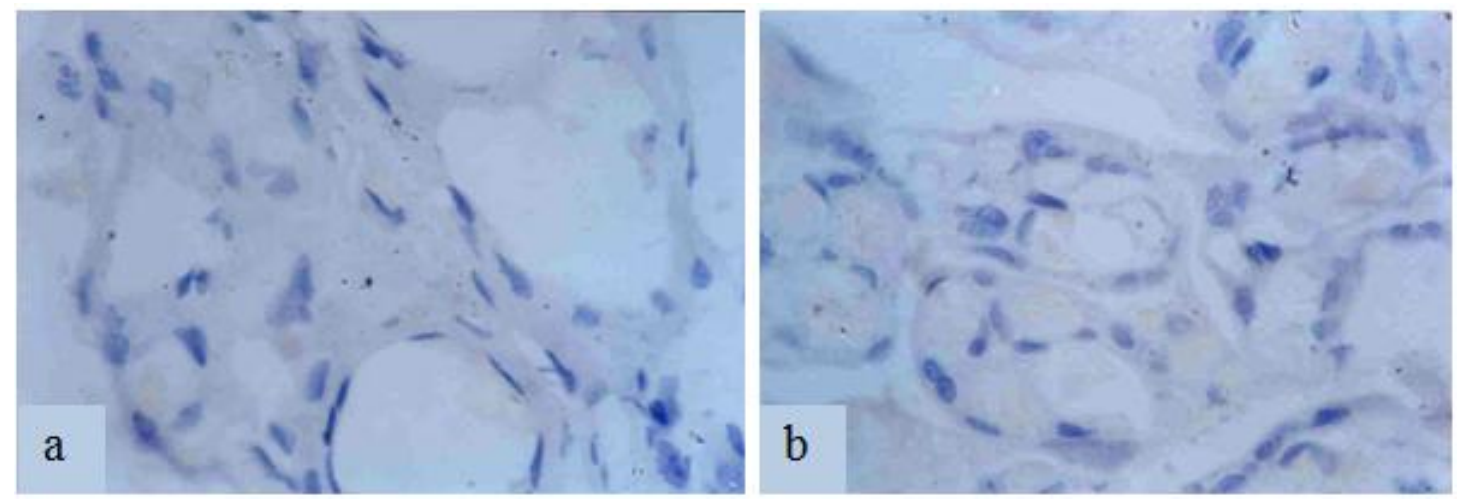

Fig3(a\&b). Negative control showing absence of immunoreactivity in endothelial cells of placenta due to elimination of primary antibodies- (a) CD31 mAb and (b) ET-1 mAb. (100x)

The distribution pattern of ET-1 immunoreactivity was also investigated in the placenta of active smokers and observed to be more intense as compared to non-smokers. In addition to the ET-1 immunostaining in the ECs lining the capillaries, focal immunostaining was observed in ECs lining small to medium sized blood vessels (Fig.4). When compared with the distribution pattern of CD-31 $\mathrm{mAb}$ in the serial sections of smoker's placenta, the distribution of ET-1 immunostaining followed similar pattern as in case of CD-31 (Fig.5). All the other cells constituting the placental villi, such as, syncytiotrophoblasts, cytotrophoblasts, connective tissues, stromal tissues and vascular smooth muscle cells were observed to be negative for ET-1 immunoreactivity in the study group.

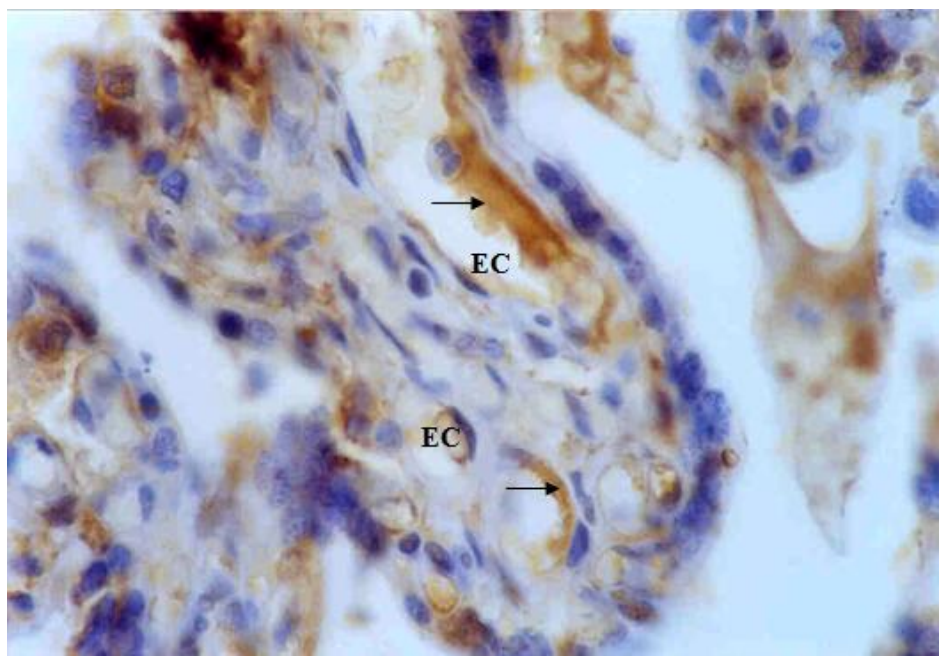

Fig4. Endothelin-1(arrow) expressing endothelial cells $(E C)$ in placental villi with intense staining. (Active Smoker, ET-1, 100x)

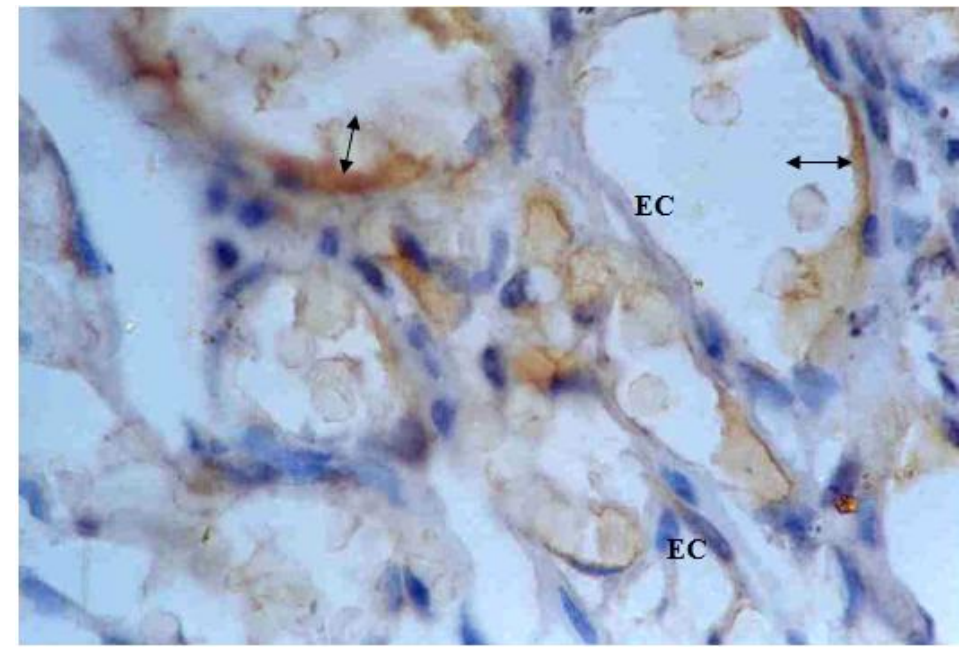

Fig5. CD-31 (double arrow) immunostaining in EC of placental villi showing similar distribution pattern as ET1 positivity in Fig.4. (Active smoker, CD-31, 100x) 


\section{DISCUSSION}

It is known that the endothelial cells lining blood vessels and capillaries in the placenta synthesize ET-1 in vivo (van Papendrop et al., 1991). In conscious rats, it is also reported that ET-1 may enhance the vascular permeability of placenta (Sirois et al., 1992). Furthermore, Svane et al. (1993) noticed that Endothelin constricts feto-placental vasculature by acting on high-affinity receptor sites. According to Haegerstrand et al. (1989), Endothelin may play a role in the constriction of umbilical vessels at the time of delivery. Therefore, it is presumed that the presence of this vasoconstrictor system in human feto-placental circulation may have a possible physiological role in the regulation of blood flow and vascular permeability in the feto-placental vascular bed.

Endothelin levels have been reported to be elevated in the maternal blood of hypertensive pregnancies as compared with normal pregnancies and are believed to play a role in vasoconstriction of fetoplacental circulation in complicated pregnancies (Mastrogiannis et al., 1991; Nova et al., 1991; Schiff et al., 1992). Kozuka et al. (1989) and Wolff et al. (1993) have also observed that ET-1 increases the myometrial smooth muscle tone, which implies that this peptide may initiate the normal parturition, as well as pre-term labor in mothers exposed to tobacco.

The present study revealed that there was no difference in the distribution pattern of ET-1 and CD-31 immunoreactivity and the immunostaining pattern of ET-1 was similar to that of endothelial cell markers, CD-31 in the placental tissues of both the study groups, viz. active smoker and non smoker group, suggesting that cells lining the venules and capillaries of placental villi can synthesize ET-1 or ET-1 like material. The ET-1 immunoreactivity, however was absent in the negative control slides of both the study groups, which proves that there was no non-specific binding of ET-1 to the placental tissues. It is thus, concluded that the ET-1 peptide is present only at those sites where endothelial cells are located along the lumen of fetal capillaries of placental villi, irrespective of control or study group.

In case of smokers and non-smokers placenta immunostaining of ET-1 was not continuous around the lumen of the endothelial cells. Expression of Endothelin-1 m-RNA in porcine and human tissue has also been described to be focal (Nunez et al. 1990). Similarly, Wilkes et al. (1993) observed focal immunostaining in the medium to large arteries and veins but noted that staining reaction was stronger in the capillaries of the placental villi. In our study, we also observed such strong immunoreactivity attributable to placental villi of active group as compared to non-smokers.

According to Wilkes et al. (1993) endothelin causes prolonged vasoconstriction in placenta which is mediated by binding of endothelin to specific high affinity receptor sites. Onda et al. (1990) are of opinion that endothelin-3 gene for endothelin is expressed by placenta. Thus it is presumed that expression of endothelin in blood vessels of placenta of both the groups may be a regulated process depending upon the requirement of fetus and nicotine may also have an influence in endothelin secretion.

Though we cannot comment on the quantity of ET-1 in the placental villi, the present study (which is a qualitative study) has revealed that the ET-1 immunoreactivity was present in endothelial cells lining the capillaries of the placental villi of smokers as well as non-smokers. It was further noted that endothelial lining of the blood capillaries of placental villi of active smokers displayed stronger immunoreactivity in comparison to non-smokers group. Therefore, it is possible that tobacco smoke or nicotine (as it the main constituent alkaloid of tobacco) might promote the synthesis of ET-1 in placental capillaries where nutrients and gas exchange take place between mother and the fetus. Thus it could be concluded that nicotine or there may be some other vasoactive peptides or hormones present in the placenta of tobacco consuming mothers, which might be responsible for bringing out the morphological and physiological changes in the placenta (Asmussen, 1980; Jauniaux and Burton, 1992).

\section{CONCLUSION}

The expression of ET-1 was observed along the endothelial lining of fetal blood vessels of smokers as well as non-smokers placenta. The immunostaining of the endothelial cells of capillaries of smokers and non-smokers placenta was not continuous. The ET-1 immunoreactivity in placenta of active smokers was consistently observed to be more intense as compared to non-smokers. Thus, it may be concluded that tobacco (nicotine) or some other vasoactive peptides or hormones present in the placenta of tobacco consuming mothers, might be responsible for bringing out the morphological and physiological changes in the placenta. 


\section{ACKNOWLEDGEMENT}

The studies received financial support by Grants from Indian Council of Medical Research (ICMR), New Delhi. The authors gratefully acknowledge the assistance of the staff at the ICMR-National Institute of Pathology, Safdarjung Hospital Campus, New Delhi and also for the financial assistance provided by the Institute.

\section{REFERENCES}

[1] Masaki T, Yanagisawa M. (1992). Endothelins. Essays Biochem. 27: 79-89.

[2] Clausen HV, Grupe Larsen L, Jørgensen A, Bzorek M (2007). The human placenta from heavy smokers: evaluation of vasoactive peptides by immunohistochemistry. APMIS. 115: 22-9.

[3] Yanagisawa M, Kurihara H, Kimura S, Tomobe Y, Kobayishi Y, Mitsui Y, Yazaki Y, Goto K, Masaki T. (1988). A novel potent vasoconstrictor peptide produced by vascular endothelial cells. Nature. 332: 411-5.

[4] Gude NM, King RG, Brennecke SP. (1991). Endothelin: release by and potent constrictor effect on the fetal vessels of human perfused placental lobules. Reprod Fertile Dev. 3: 495-500.

[5] Myatt L, Brewer AS, Brockman DE. (1992). The comparative effects of big endothelin-1, endothelin-1 and endothelin-3 in the human fetal placental circulation. Am J Obstet Gynecol. 167: 1651-6.

[6] Sudo N, Kamoi K, Ishibashi M, Yamaji T. (1993). Plasma endothelin-1 and big endothelin-1 levels in women with pre-eclampsia. Acta Endocrinol (Copenh). 129: 114-20.

[7] Marinoni E, Picca A, Scucchi L, Cosmi EV, Di Iorio R. (1995). Immuno histochemical localization of endothelin-1 in placenta and foetal membranes in term and pre-term human pregnancy. Am J Reprod Immunol. 34: 213-8.

[8] Hemsen A, Gillis C, Larsson O, Haegerstrand A, Lundberg JM. (1991). Characterization, localization and actions of endothelins in umbilical vessels and placenta of man. Acta Physiol Scand. 143: 395-404.

[9] Malassine A, Croneir L, Mondon F, Mignot TM, Ferre F. (1993). Localization and production of immunoreactive endothelin-1 in the trophoblast of human placenta. Cell Tissue Res. 271: 491-7.

[10] Economides D and Braithwaite J. (1994). Smoking, pregnancy and fetus. J R Soc Health. 114: 198-201.

[11] Asmussen I. (1980). Ultra-structure of the villi and fetal capillaries in placentas from smoking and nonsmoking mothers. Br J Obstet Gynaecol. 87: 239-45.

[12] Jauniaux E, Burton GJ. (1992). The effect of smoking in pregnancy on early placental morphology. Obstet Gyneacol. 79: 645-8.

[13] van Papendrop CL, Cameron IT, Davenport AP, King A, Barker PJ, Huskisson NS, Gilmour RS, Brown MJ, Smith SK. (1991). Localization and endogenous concentration of endothelin-like immuno-reactivity in human placenta. J Endocrinol. 131: 507-11.

[14] Sirois MG, Filep JG, Rousseau A, Fournier A, Plante GE, Sirois P. (1992). Endothelin-1 enhances vascular permeability in conscious rats, role of thromboxane A2. Eur J Pharmacol. 214: 119-25.

[15] Svane D, Larsson B, Alm P, Andersson KE, Forman A. (1993). Endothelin-1: immuno-cytochemistry, localization of binding sites, and contractile effects in human uteroplacental smooth muscle. Am J Obstet Gynecol. 168: 233-41.

[16] Haegerstrand A, Hemsen A, Gillis C, Larsson O, Lundberg JM. (1989). Endothelin: Presence in human umbilical vessels, high levels in fetal blood and potent constrictor effect. Acta Physiol Scand. 137: 541-2.

[17] Mastrogiannis DS, O'Brien WF, Krammer J, Benoit R. (1991). Potential role of endothelin-1 in normal and hypertensive Pregnancies. Am J Obstet Gynecol. 165: 1711-6.

[18] Nova A, Sibai BM, Barton JR, Mercer BM, Mitchell MD. (1991). Maternal plasma levels of endothelin is increased in pre-eclampsia. Am J Obstet Gynecol. 165: 724-7.

[19] Schiff E, Ben-Baruch G, Peleg E, Rosenthal T, Alcalay M, Devir M, Mashiach S. (1992). Immunoreactive circulating endothelin-1 in normal and hypertensive pregnancies. Am J Obstet Gynecol. 166: 6248.

[20] Kozuka M, Ito T, Hirose S, Takahashi K, Hagiwara H. (1989). Endothelin induces two types of contractions of rat uterus: phasic contractions by way of voltage-dependent calcium channels and developing contractions through a second type of calcium channels. Biochem Biophys Res Commun. 159: 317-23.

[21] Wolff K, Nisell H, Modin A, Lundberg JM, Lunell NO, Lindblom B. (1993). Contractile effect of endothelin 1 and endothelin 3 on myometrium and small intra-myometrial arteries of pregnant women at term. Gynecol Obstet Invest. 36: 166-71.

[22] Nunez DJ, Brown MJ, Davenport AP, Neylon CB. Schofield JP, Wyse RK. (1990). Endothelin-1 m-RNA is widely expressed in porcine and human tissues. J Clin Invest. 85: 1537-41. 
[23] Wilkes BM, Susin M, Mento PF. (1993). Localization of endothelin-1-like immunoreactivity in human placenta. J Histochem Cytochem. 41: 535-41.

[24] Onda H, Ohkubo S, Ogi K, Kosaka T, Kimura C, Matsumoto H, Suzuki N, Fujino M. (1990). One of the endothelin gene family, endothelin 3 gene, is expressed in the placenta. FEBS Lett. 261: 327-30.

[25] Driscoll SG, Langston C (1991). College of American Pathologists Conference XIX on examination of the placenta: report of the working group on methods for placental examination. Arch Pathol Lab Med. 115: 704-8.

[26] Barros JS, Bairos VA, Baptista MG, Fagulha JO (2001). Immunocytochemical localization of endothelin-1 in human placenta from normal and preeclamptic pregnancies. Hypertens Pregnancy. 20: 125-37.

[27] Salafia C, Shiverick K (1999). Cigarette smoking and pregnancy II: vascular effects. Placenta. 20: 273-9.

[28] Quinton AE, Cook CM, Peek MJ (2008). The relationship between cigarette smoking, endothelial function and intrauterine growth restriction in human pregnancy. BJOG. 115(6): 780-4.

[29] Gude NM, Roberts CT, Kalionis B, King RG (2004). Growth and function of the normal human placenta. Thromb Res. 114(5-6): 397-407.

[30] To WKL. (2012). A role of ATP in modulating vasomotion during hypoxia in umbilical cord blood vessels (Doctoral dissertation, University of Birmingham).

[31] Carter AM, Myatt L. (1995). Control of placental blood flow: workshop report. Reprod Fertil. 7: 1401-6.

[32] Hsu SM, Raine L, Fanger H. (1981). Use of avidin-biotin-peroxidase complex (ABC) in immunoperoxidase techniques: a comparison between $\mathrm{ABC}$ and unlabeled antibody (PAP) procedures. $\mathrm{J}$ Histochem Cytochem. 29(4): 577-80.

Citation: B. Bastia et al., "Expression of Endothelin-1 in Human Placenta of Active Smokers: An Immunohistochemical Study", International Journal of Research Studies in Biosciences (IJRSB), vol. 5, no. 12, pp. 34-40, 2017. http://dx.doi.org/10.20431/2349-0365.0512005

Copyright: () 2017 Authors. This is an open-access article distributed under the terms of the Creative Commons Attribution License, which permits unrestricted use, distribution, and reproduction in any medium, provided the original author and source are credited. 